\title{
Uma Proposta de Uso de Agentes Inteligentes para auxiliar a Criação de Sub-comunidades de Aprendizagem na CV- Muzar $^{1}$
}

\author{
Cristiane Durigon Testa ${ }^{2}$ - 57547@ lci.upf.br \\ Ana Carolina Bertoletti De Marchi ${ }^{3}$ - Carolina@upf.br \\ Flávia Biondo da Silva ${ }^{4}$ - biondo@upf.br
}

Resumo: Com o avanço dos meios de comunicação digitais muitos conceitos sofreram mudanças de significado. Um exemplo disso é o significado de comunidade, que antes era associado a um determinado lugar. Hoje as comunidades ultrapassam todas as barreiras de tempo e lugar, aproximando casa vez mais o aqui e agora. Diante disso, surgiram os ambientes de comunidades virtuais, os quais têm como objetivo reunir pessoas de interesses em comum a fim de trocar conhecimento e experiências de forma colaborativa por meio de mecanismos de comunicação síncronos e assíncronos. Essas comunidades assumiram uma interdependência social que fez surgir à possibilidade de se criarem novas comunidades ou subdivisões em grupos menores conhecidos como sub-comunidades. Neste artigo iremos apresentar uma proposta de aplicação de Agentes Inteligentes para auxiliar os participantes na criação das sub-comunidades e para a realização de pesquisas.

Palavras-chaves: Comunidade Virtual, Aprendizagem Colaborativa, Agentes Inteligentes e Sub-comunidades.

A Proposal of Use of Intelligent Agents to assist the Creation of Sub-communities of Learning in the CV-Muzar

Summary: With the advance of the digital medias many concepts had suffered changes from meaning. An example of this is the community meaning, that before was associated to one definitive place. Today the communities exceed all the barriers of time and place, approaching house time more here and now. Ahead of this, the environments of virtual communities had appeared, which have as objective to congregate people of interests in common in order to change to knowledge and experiences of colaborativa form by means of synchronous and asynchronous mechanisms of communication. These communities had assumed a social interdependence, that made to appear the possibility of if creating new communities or subdivisões in known lesser groups as sub-communities. In this article we will go to present a proposal of application of Intelligent Agents to assist the participants to create sub-communities and for the accomplishment of research.

\footnotetext{
${ }^{1}$ Artigo elaborado a partir do trabalho de conclusão do curso de Ciência da Computação e integrante do projeto de pesquisa "Informática auxiliando aprendizagem em museus de ciências e tecnologia, através de padrão de metadados para objetos de aprendizagem", apoiando pelo CNPq e Fapergs.

${ }^{2}$ Bolsista de Iniciação Científica e aluna do Curso de Ciência da Computação/UPF. 57547@lci.upf.br.

3 Professora doutora em Informática na Educação (PGIE/UFRGS), professora e pesquisadora da UPF, carolina@upf.br.

${ }^{4}$ Coordenadora do MUZAR e mestre em Educação pela Universidade de Passo Fundo, biondo@upf.tche.br. V. $4 \mathrm{~N}^{\mathrm{o}}$ 1, Julho, 2006
} 
Word-keys: Virtual community, collaborate Learning, Agents Intelligent and Subcommunities.

\section{Introdução}

Nos dias de hoje a Internet não é mais vista apenas como um ambiente de intensa fonte de informação, mas, também, como um meio de comunicação que propicia a formação de grupos sociais mediados pelo computador.

Muitos são os serviços oferecidos na rede que facilitam a comunicação interpessoal e, conseqüentemente, a formação de grupos com interesses em comum. Há tempos atrás, a formação de grupos era considerada um fator relevante para o desenvolvimento da comunidade. Assim, pessoas que possuíam interesses em comum formavam grupos e comunidades a fim de buscar conhecimentos que os diferenciava dos demais grupos. Neste período, as comunidades formavam-se com base em um local determinado, fazendo com que o significado de comunidade assumisse, muitas vezes, sinônimo dependente de um lugar, de um determinado espaço e de uma dimensão geográfica.

Com o advento da comunicação eletrônica, foram excluídas todas as dimensões do aqui e agora, ou seja, a existência de uma comunidade não ficou restrita apenas as dimensões tempo e espaço. Diante desse novo contexto, passaram a surgir os grupos sociais mediados por computador, os quais são conhecidos como Comunidades Virtuais (CVs).

As CVs possibilitam que seus participantes, através de meios de comunicação on-line, enviem mensagens assíncronas e síncronas, proporcionando discussões, trocas de conhecimento e possibilitando a aprendizagem de forma colaborativa, por meio da troca constante de informações pelos participantes ativos.

Com os avanços tecnológicos e cientifico, muitos significados que tínhamos como concretos, como, por exemplo, o termo comunidade, passou a assumir um enfoque muito mais complexo em termos de gerenciamento e trocas, já que o número de participantes nesses grupos aumentam exponencialmente.

As comunidades assumiram uma interdependência social, que fez surgir a possibilidade de se criarem novas comunidades ou subdivisões em grupos menores conhecidos como sub-comunidades.

As sub-comunidades são parte integrante de um grupo maior chamado "comunidade". Muitas vezes um grande grupo é dividido em pequenos grupos para executar tarefas, discutir e avaliar trabalhos de um determinado assunto de interesse, promovendo assim um processo de colaboração com o grupo maior.

Segundo Palloff e Pratt (2002, p. 83), os grupos menores são necessários se os trabalhos tiverem de ser escritos conjuntamente ou se a apresentação on-line tiver de ser preparada, por exemplo, para mostrar ao grupo maior.

A possibilidade e necessidade de se criar grupos menores para promover discussões mais pontuais, foi constatada na Comunidade Virtual do Museu Zoobotânico Augusto Ruschi da UPF (CV-Muzar). A CV-Muzar (De Marchi, 2005) foi desenvolvida para armazenar todas as informações contidas nos museus, facilitando, assim, o relacionamento e a busca de informações por grupos de pessoas que se interessam pela área de ciências naturais. A CV-Muzar foi desenvolvida especificamente para aproximar o seu público das inúmeras informações e peças museológicas disponíveis no ambiente e que antes ficavam restritas para acesso local.

Este artigo tem como objetivo apresentar brevemente a CV-Muzar e mostrar as 
constatações atingidas por meio de uma experimentação realizada e que levaram a equipe a definição de novos requisitos a serem implementados. Esses novos requisitos estão relacionados à criação de sub-comunidades dentro da CV-Muzar, mediadas por Agentes Inteligentes responsáveis por auxiliar os participantes na criação desses grupos menores.

Em um primeiro momento, iremos apresentar o ambiente CV-Muzar e suas funcionalidades. A seguir será descrita a idéia da formação e desenvolvimento das subcomunidades e, por fim, mostraremos a proposta de se aplicar agentes inteligentes para auxiliar a criação das sub-comunidades.

\section{Um pouco sobre a CV-Muzar e sua experimentação}

A CV-Muzar (De Marchi, 2005) é composta por dois módulos principais: o módulo que visa apoiar as atividades didáticas, oferecendo ao visitante acesso a um conjunto de ferramentas de comunicação; e o módulo de gerência do repositório de recursos didáticos, onde são disponibilizados todos os objetos de aprendizagem disponíveis.

Os OAs são elementos da aprendizagem apoiada pelo computador e que possuem como base o paradigma de Orientação à Objetos da Ciência da Computação, tendo como principal caracteristica a possibilidade de reutilizar os componentes (objetos) em diferentes contextos. Muitos OAs tiveram que ser desenvolvidos com base na documetação existente no Museu. Mais de $90 \%$ dessa documentação era em papel, o que tornou necessário a criação de pequenos módulos digitais desenvolvidos em diferentes ferramentas.

Estes OA foram inseridos no repositório de Gerenciamente de Objetos de Aprendizagem - GROA (Miranda, 2004). Este gerenciamento foi desenvolvido utilizando Zope 2.6 e Métodos Python para a recuperação dos OA. Desta forma, optamos por desenvolver o ambiente de Comunidade Virtual utilizando a mesma ferramenta. Entre as vantagens de se utilizar essa ferramenta podemos citar: a existência de produtos prontos e a filosofia OpenSource.

Além do repositório de OAs, a CV-Muzar também conta com ferramentas de pesquisa por meio de Mapa de Tópicos e ferramentas de comunicação sincrona e assincrona como chat, forum, mural, entre outras, que são ferramentas essenciasis para promover a aprendizagem informal e colaborativa.

O objetivo da CV-Muzar é promover a aprendizagem informal a partir de interesses pessoais dos membros de modo a definir uma rede de relacionamento autoorganizada, visando os objetivos comuns que os levem a aprendizagem contínua e permanente.

As figuras abaixo ilustram o ambiente de comunidade virtual e suas ferramentas. A figura 1 mostra uma visão geral do ambiente e suas funcionalidades. 


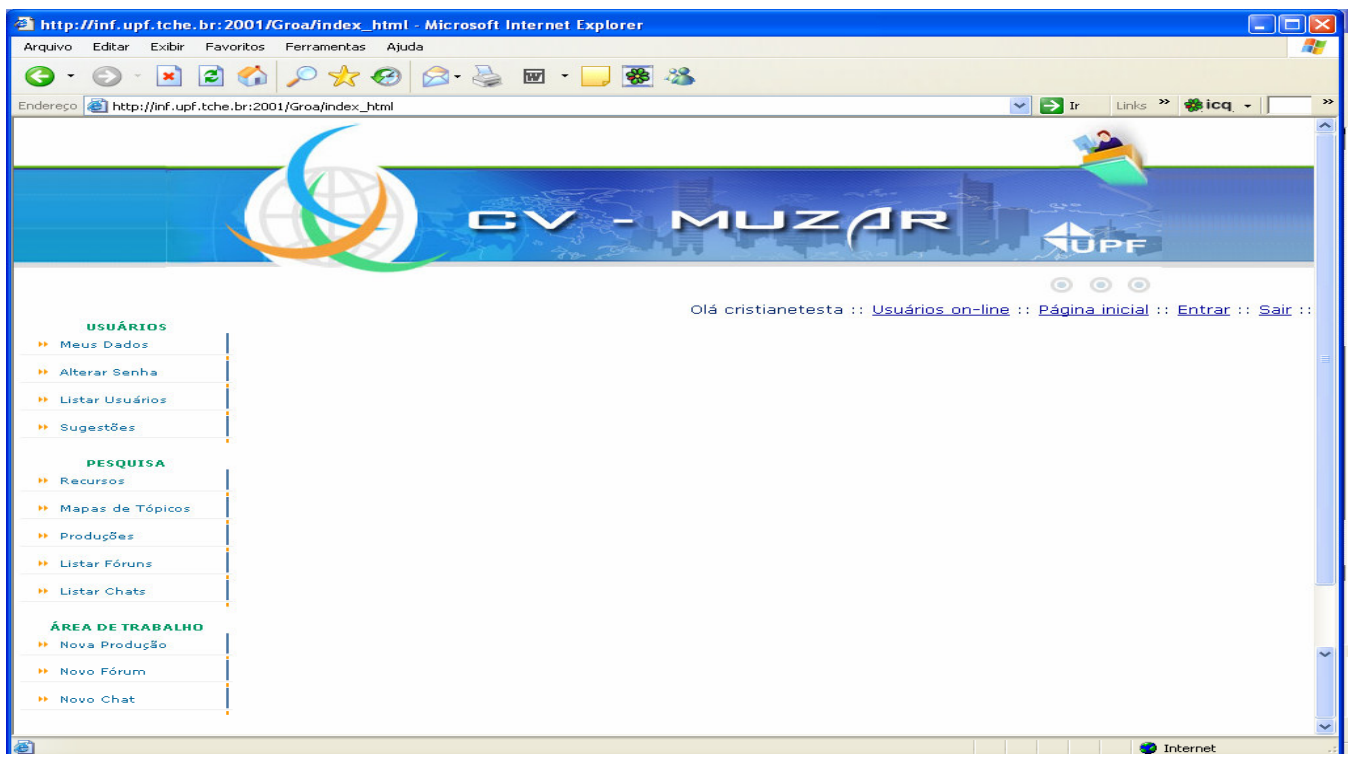

Figura 1 - Visão Geral

De modo a verificar o quanto a CV-Muzar incentiva à participação/envolvimento do visitante em atividades de aprendizagem informal com relação à efetividade da produção dos mesmos, foi realizada uma avaliação com um grupo de pessoas convidadas (para maiores detalhes ver De Marchi, 2005)

Com essa avaliação foi possível identificar os meios pelos quais se pode motivar os visitantes a produzirem novos conhecimentos durante sua visita informal ao museu. Neste sentido, as salas de bate-papo foram apontadas como a melhor maneira de promover trocas e possibilitar a colaboração entre os participantes. Entretanto, para um melhor aproveitamento dessa ferramenta é desejável que sejam marcadas reuniões pontuais com grupos pequenos, para que se possa encaminhar discussões ricas onde o ambiente se assuma como um espaço discursivo envolvendo o participante em atividades de aprendizagem informal. Complementando, foi salientado por alguns participantes, não como forma de desaprovação, mas sim visando facilitar a navegação, que a riqueza de informações presente no ambiente, causa, por vezes, uma sobrecarga de informações. Como forma de sanar este problema foi sugerida a criação de grupos menores onde se torne viável promover discussões mais especificas sobre o assunto geral da Comunidade. Esses grupos menores definidos como sub-comunidades, serão abordados na próxima seção.

\section{Comunidades Virtuais e Sub-comunidades}

Comunidades virtuais são definidas por Pallof e Pratt (2002) como comunidades que usam as tecnologias de rede, especialmente a Internet, para estabelecer a comunicação além das barreiras geográficas e de tempo. Em comparação as comunidades tradicionais, as comunidades virtuais na Web diferem em vários pontos. As comunidades tradicionais possuem lugares fixos e dimensionais, e seus participantes são aceitos através de normas. A dinâmica do grupo, geralmente, sobrepõe-se à 
expressão individual. Em contrapartida, nas comunidades virtuais a identificação se dá

em torno de uma idéia ou trabalho, não importando, lugar, espaço e tempo. Dentro das comunidades virtuais também são definidas normas, mas não são dominantes como nas comunidades tradicionais.

As comunidades virtuais de aprendizagem também são utilizadas para prover um meio de comunicação e troca de informações entre participantes que possuem interesses em comum, proporcionando uma comunicação interativa que leva a aprendizagem continua e permanente.

Diante desta informação e também com a experiência que tivemos na CVMuzar, podemos afirmar que é muito importante tratar o tamanho do grupo. Um dos motivos de se preocupar com o tamanho do grupo é que um ambiente de comunidade virtual é um ambiente amplo com inúmeras informações circulando e sendo inseridos a todo o momento, fazendo com que os participantes se sintam perdidos diante tanta informação. Assim sendo, com a criação de grupos menores, os membros podem participar de grupos que tratam de assuntos específicos e que atendam a sua área de interesse.

Ainda sendo, os grupos menores são necessários se os trabalhos tiverem de ser escritos conjuntamente ou se a apresentação on-line tiver de ser preparada, por exemplo, para mostrar ao grupo maior (Pallof ePratt, 2002).

Para a criação destas sub-comunidades dentro da CV-Muzar, iremos aplicar Agentes Inteligentes, que terão como objetivo auxiliar a criação e a interação destes sub-grupos. Desta maneira, um agente pode auxiliar o participante a criar um sub-grupo por meio de monitoração e atuação de forma autônoma, podendo ajudar a cumprir tarefas na formação do grupo e das ferramentas que serão necessárias para a comunicação e troca de conhecimento entre os participantes.

A proposta de desenvolver sub-comunidades partiu da necessidade dos participantes ativos na comunidade poderem criar projetos, ou até mesmo, cursos específicos de um determinado assunto de interesse de um grupo menor. Assim, um participante poderá criar a uma nova área na comunidade, passando o mesmo a assumir o papel de coordenador do grupo, podendo escolher que ferramentas serão necessárias para o desenvolvimento do curso.

As ferramentas disponíveis para as sub-comunidades são tanto de natureza síncrona e assíncrona. Como ferramentas síncronas podemos citar Chat e mensagens instantâneas as quais podem ser utilizadas pelos participantes para troca de conhecimento em tempo real, debates sobre os assuntos que circulam dentro do grupo e também durante a participação de algum curso que estará ocorrendo dentro das subcomunidades, podendo desta forma aproximar os participantes através das conversações. Pois neste momento as idéias de cada componente dos grupos são expostas e debatidas por todos. Já para exemplificar ferramentas assíncronas temos fórum, mural, notícias e wiki, muito utilizadas para o andamento das discussões do grupo sendo uma fonte estimuladora para os participantes interagiram mais nos assuntos inseridos ao grupo e reguladora das ações individuais. (Piaget 1998, p.148-149 em tal) Segundo o autor, relata que as atividades em grupo são consideradas como uma forma de facilitar o processo de aprendizagem. Quando um participante atua em uma coletividade a sua forma de expor seu pensamento, renuncia aos interesses individuais, passando a pensar em função do coletivo. Para alcançar o objetivo de aprendizagem colaborativa é preciso disponibilizar ferramentas apropriadas para incentivar a construção coletiva e uma das ferramentas que se encaixa neste perfil são as ferramentas de wiki que atribui em sua essência grande parte dos requisitos. 
A ferramenta de wiki terá dupla função nas sub-comunidades, pois pode ser utilizada para a construção de textos como uma forma de organização dos assuntos gerados dentro do grupo e também como uma ferramenta de produção, podendo os participantes incluir os textos produzidos coletivamente na CV-Muzar Geral como contribuição (produção) dos usuários. Ficando está produção disponível no repositório das produções tanto para visualização quanto a recuperação e utilização deste por qualquer outro grupo o participante atuante no ambiente da comunidade.

A Figura abaixo ilustra como é o funcionamento na CV-Muzar com a criação das sub-comunidades e a ação dos Agentes Inteligentes.

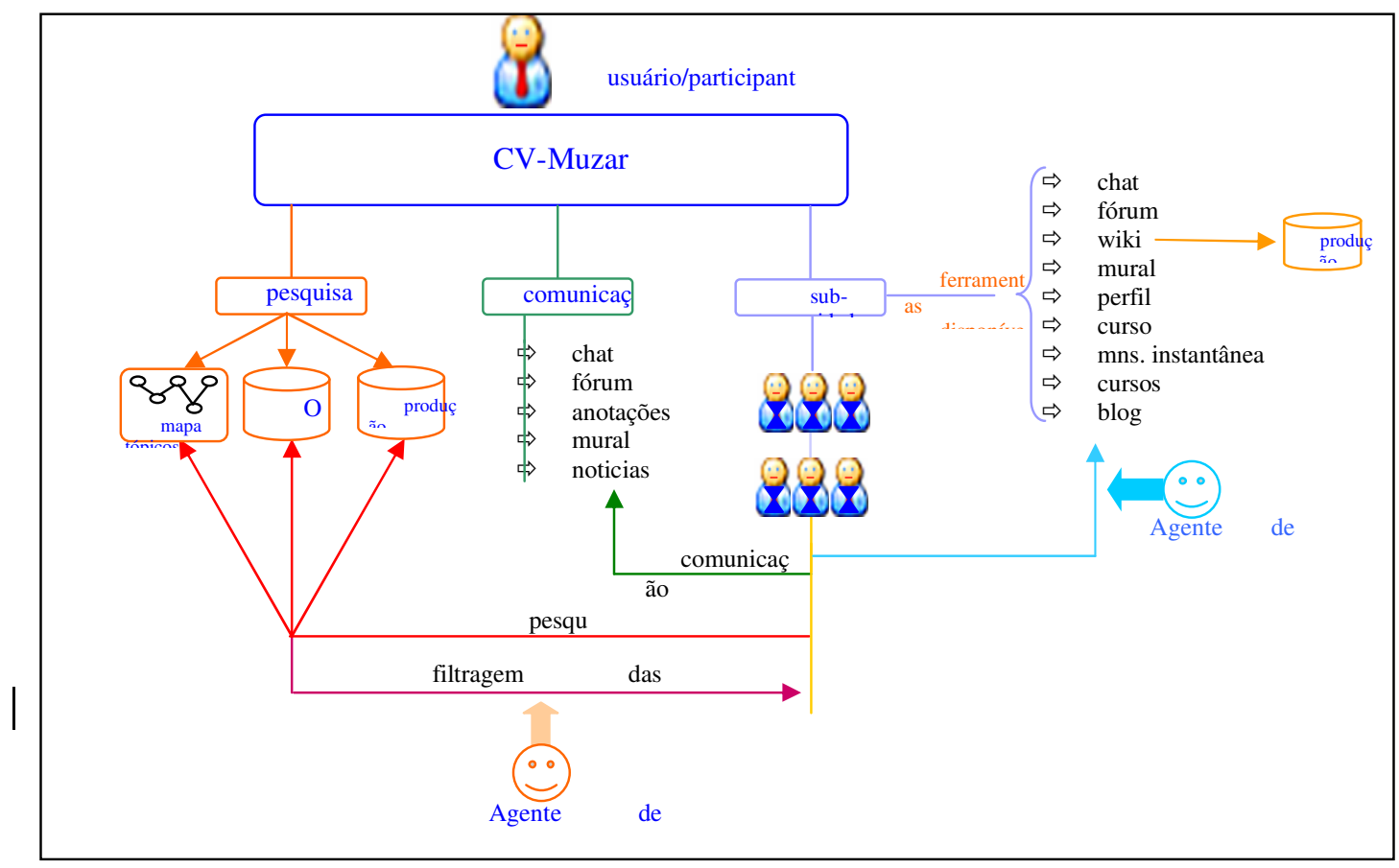

Figura 2 - Arquitetura CV-Muzar e Sub-comunidades

\subsection{O Papel dos Agentes Inteligentes}

O termo Agentes Inteligentes surgiu em meados dos anos 80 com o advento das pesquisas na área de Inteligência Artificial e a partir dali muitos autores passaram a conceituar o termo. Bogo (2003) apresenta alguns destes conceitos:

- Agentes Inteligentes são entidades reais ou abstratas capazes de agir sobre ela mesma ou sobre seu ambientes. Ferber(1991) citado por Bogo(2003).

- São sistemas computacionais residentes em ambientes dinâmicos complexos, os quais percebem e atuam autonomamente, e, ao fazê-lo, realizam um conjunto de objetivos e tarefas para os quais foram designados. Souza(2001) citado por Bogo(2003). 
Entretanto, atualmente, o mais aceito é que agente é um programa de software que auxilia o usuário na realização de alguma tarefa ou atividade. Wooldridge(2002) citado por $\operatorname{Bogo}(2003)$.

A tecnologia de agentes mostra-se muito flexível para a resolução de problemas dinâmicos e vem sendo aperfeiçoada para a aplicação em ambientes virtuais de aprendizagem.

Os agentes podem existir em um ambiente que contém ou não outros agentes. Em algumas situações, agentes podem executar tarefas por si próprios ou em conjunto com os demais. Isso acontece a todo o momento no mundo real, pois, quando alguém tem necessidade de resolver algum problema que envolva algum conhecimento ou habilidade que não possua, necessita do auxílio de outra pessoa, apta a fazê-lo.

Neste caso o agente pode auxiliar o participante a criar um sub-grupo por meio de monitoração e atuação de forma autônoma, podendo ajudar a cumprir tarefas na formação do grupo e das ferramentas (como ilustra a figura 3 e 4) que serão necessárias para a comunicação e troca de conhecimento entre os participantes. Abaixo segue algumas aplicações de agentes em sistemas de ambientes virtuais de aprendizagem. Cunha(2002) citado por Bogo(2004).

CALM: É um ambiente para a criação dinâmica e o gerenciamento de grupo de estudo, formado por estudantes distribuídos que compartilham um material on-line. No CALM, do pondo de vista tecnológico, a colaboração entre usuários de um ambiente de aprendizado on-line depende de duas tarefas:

- a definição e a formação do grupo: os estudantes podem estabelecer um "perfil de colaboração", indicando as características do grupo ao qual eles gostariam de participar.

- o estabelecimento (síncrono e assíncrono) de sessões de comunicação.

No CALM é definida uma arquitetura de agentes composta por um conjunto de agências que implementam o ambiente de aprendizado colaborativo. Uma agência tem a responsabilidade de obter as especificações das metas dos usuários e executar as ações necessárias para cumpri-las. Eventualmente a agência delega algumas tarefas a outros agentes que a ajudarão na execução destas.

GUARDIAN AGENT: é um conjunto de agentes desenvolvido para monitorar e assistir a estudantes que estão trabalhando em grupo, em um ambiente distribuído. O sistema apóia o trabalho em grupo em educação on-line fornecendo a cada estudante um agente que monitora o seu progresso, comparando com o plano do grupo e tomando medidas quando necessário. As medidas tomadas têm como objetivo a manutenção da coesão e da confiança do grupo em seus membros.

A tecnologia de agentes mostra-se muito flexível para a resolução de problemas dinâmicos e vem sendo aperfeiçoada para a aplicação em ambientes virtuais de aprendizagem.

Os agentes podem existir em um ambiente que contém ou não outros agentes. Em algumas situações, agentes podem executar tarefas por si próprios ou em conjunto com os demais. Isso acontece a todo o momento no mundo real, pois, quando alguém tem necessidade de resolver algum problema que envolva algum conhecimento ou habilidade que não possua, necessita do auxílio de outra pessoa, apta a fazê-lo. Wooldridge(2002) citado por BOGO (2003).

Assim sendo, neste trabalho definimos dois tipos que agentes que irão V. $4 \mathrm{~N}^{\mathrm{o}}$ 1, Julho, 2006 
interagir na criação e desenvolvimento das sub-comunidades, os quais definimos como Agente de Apoio e Agente de Pesquisa. O Agente de Apoio tem a função de auxiliar o participante criador do grupo na escolha das ferramentas necessárias para a comunicação e troca de conhecimento entre os participantes. Já o Agente de Pesquisa tem como função filtrar as informações contidas na CV-Muzar Geral para a subcomunidade, conforme o assunto que esta irá tratar.

Quando o usuário/participante decidir por criar uma sub-comunidade, o mesmo terá que executar/passar por todo um processo de criação e definição de ferramentas que irão auxiliar na comunicação, colaboração e troca de conhecimento.

Neste ponto é que entra o trabalho do Agente Inteligente ao qual designamos como Agente de Apoio. Nesta etapa o participante assume o papel de coordenador do grupo menor. O coordenador tem como função escolher as ferramentas disponíveis no painel de acordo com a necessidade do grupo. Enquanto o coordenador for escolhendo as ferramentas, o Agente de Apoio vai auxiliá-lo no sentido de informar as características de cada ferramenta e como os participantes podem tirar proveito desta. Por exemplo, se o coordenador escolher a ferramenta de chat, o Agente de Apoio vai informar às características da sala de bate-papo, funcionalidades dentro do grupo, número de participantes para conversação livre e número limite de participantes durante o curso proposto.

Além do Agente de Apoio, fica disponível o Agente de Pesquisa que tem como principal função filtrar os objetos de aprendizagem disponível no repositório de OA na CV-Muzar e no repositório de Produção da CV-Muzar referente às áreas de conhecimento da sub-comunidade.

Este Agente de Pesquisa pode ser ativado ou não na comunidade dependendo da necessidade e da característica das sub-comunidades. Esta filtragem de informações será uma imagem dos OA e Produções contidas na CV-Muzar. Na hora da utilização e recuperação dos OAs, o acesso será no repositório da CV-Muzar e refeita a imagem dentro da sub-comunidade. As imagens são refeitas pelo principal motivo de não deixar os participantes perdidos diante da grande quantidade de informações disponibilizadas na comunidade geral. Assim, é possível organizar as informações de maneira que os participantes possam realizar as suas pesquisas e receber as informações somente do assunto requisitado.

Desta forma, este trabalho tem como objetivo aproximar mais os participantes por áreas de interesse e, também, intensificar a participação e a geração de conhecimento, uma vez que, muitas vezes, os participantes podem sentir-se perdidos dentro da comunidade pelo fato do grande fluxo de informações. A formação de subcomunidade pode intensificar e incentivar mais a participação ativa dos participantes tanto na CV-Muzar quanto nas sub-comunidades. 


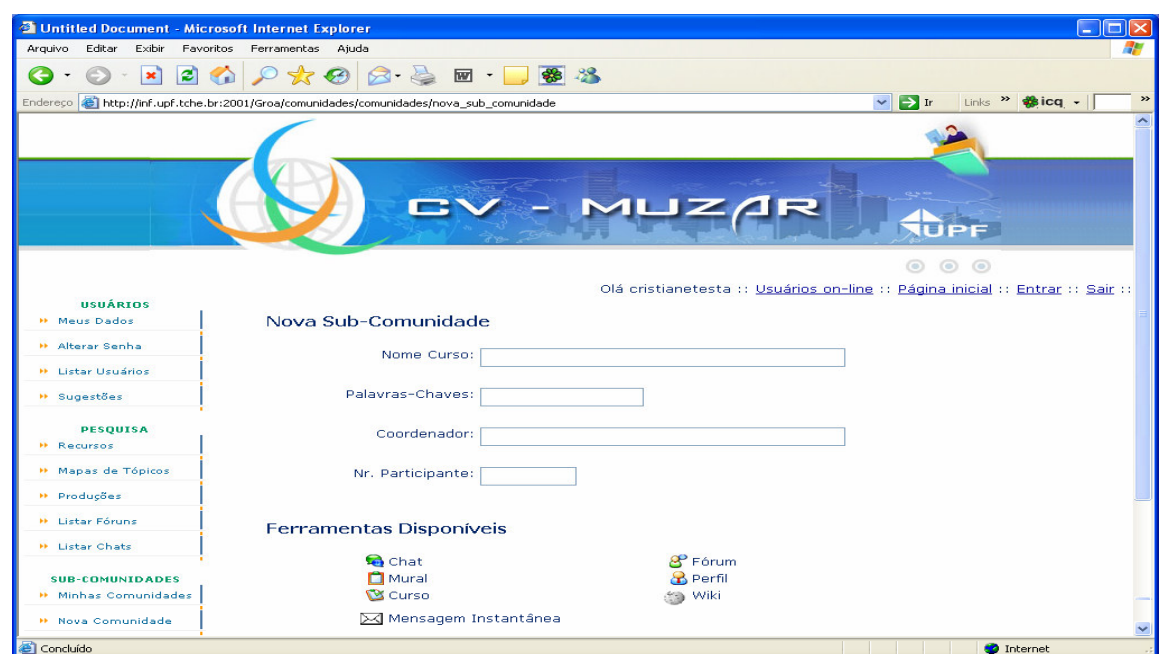

Figura 3 - Nova Sub-Comunidade

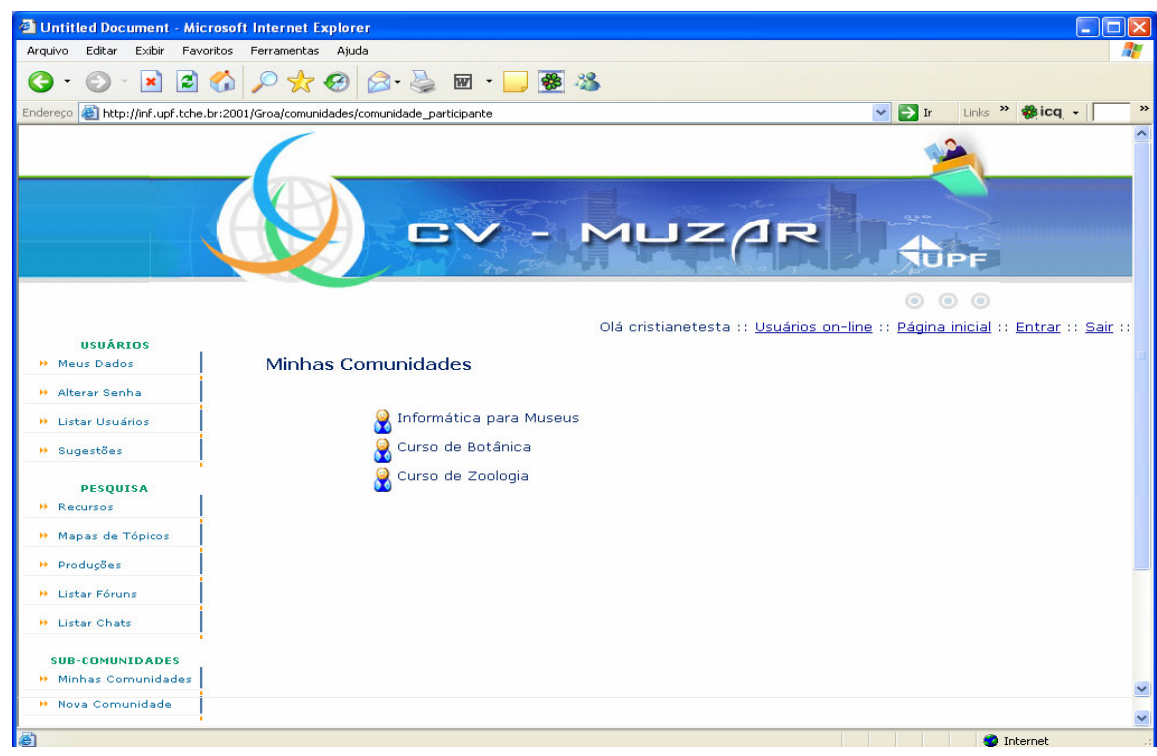

Figura 4 - Minhas Sub-Comunidades

\section{Conclusão}

Com o desenvolvimento das sub-comunidades dentro da CV-Muzar estaremos proporcionando uma centralização de assuntos através da criação dos cursos. Promovendo assim, uma maior troca de informação entre os participantes e incentivando para o conhecimento colaborativo e informal através dos recursos de OA e produções dos usuários disponíveis na comunidade.

Adicionalmente, a aplicação de Agentes Inteligentes irá facilitar a criação das

sub-comunidades, pois os mesmos terão como função guiar o coordenador da subcomunidade a escolher as ferramentas necessárias para participação ativa do grupo, troca de informação propiciada através do uso das ferramentas e a filtragem de informação referente a área de interesse do grupo. 
As sub-comunidades, além de proporcionar aos participantes uma melhor organização das informações e ainda poderão contribuir com a CV-Muzar Geral através da produção de textos coletivos utilizando para isso a ferramenta wiki, formando assim novas fontes de pesquisa que poderão ser reutilizadas por toda a comunidade.

Assim podemos concluir que é de grande valia os ambientes de comunidades virtuais e a proposta da criação dos grupos menores enriquecendo a CV-Muzar que vai poder ter uma maior abrangência dos assuntos separando-os conforme os interesses dos participantes com o objetivo comum de gerar aprendizagem.

\section{Referências Bibliográficas}

BERTOLETTI-DE-MARCHI, Ana Carolina; TESTA, Cristiane Durigon; COSTA, Antônio Caros da Rocha. Um ambiente de comunidade virtual baseado em objetos de aprendizagem para apoiar a aprendizagem em museus. Renote Revista Novas Tecnologias na Educação, Porto Alegre, 2005.

BOGO, Luís Henrique. Criação de Comunidades Virtuais a partir de Agentes Inteligentes: Uma aplicação em E-learning, 2003. Dissertação (Mestrado em Engenharia de Produção) - PPGEP, UFSC. Florianópolis, 2003.

CASTELLS, Manuel. A Sociedade em Rede. 9. ed. São Paulo: Paz e Terra, 1999. 698p.

DE MARCHI, A. C. B. Um ambiente de suporte a comunidades virtuais baseados em repositório de objetos de aprendizagem informal em museus. 2005. Tese (Doutorado em Informática em Educação) - PGIE, UFRGS. Porto Alegre, 2005.

FAVERO, Luis Elói; BRITO, Silvana Rossy de; SILVA, Aleksandra do Socorro da. Modelo baseado em agentes para acompanhamento do processo de aprendizagem em ambientes virtuais.

HAETINGER, Daniela; ABEGG, Ilse, COSTA, Janete, TAROUCO Liane. TWiki, uma ferramenta de co-autoria livre. Acessado em 04 jun. 2006. Disponível em: http://www.cinted.ufrgs.br/renote/nov2005/artigosrenote/a77_TWiki.pdf.

LÉVY, P. Cibercultura. São Paulo: Editora 34, 1999.

LÉVY, P. O que é Virtual. São Paulo: Editora 34, 1998.

MIRANDA, R. GROA - Um Sistema de Gerencia de Repositórios de Objetos de Aprendizagem, 2004. Dissertação (Mestrado em Ciência da Computação) - PPGCC, UFRGS. Porto Alegre, 2004.

PALLOFF, R.M. e PRATT, K. Construindo Comunidades de Aprendizagem no

Ciberespaço: Estratégias eficientes para salas de aula on-line. Trad. Vinícius Figueira. Porto Alegre: Artmed, 2002. 248p.

TESTA, Cristiane Durigon; BERTOLETTI-DE-MARCHI, Ana Carolina; COSTA, Antônio Caros da Rocha. Desenvolvimento e Funcionamento de uma Comunidade Virtual para Museus com acesso a Repositório de Objetos de Aprendizagem. Hífem, Uruguaiana, 2005. 